\title{
Olive oil, an essential component of the Mediterranean diet, and breast cancer
}

\author{
Eduard Escrich*, Raquel Moral and Montserrat Solanas \\ Department of Cell Biology, Physiology and Immunology, Physiology Unit, Medicine School, \\ Universitat Autònoma de Barcelona, 08193 Bellaterra, Barcelona, Spain
}

Submitted 13 May 2011: Accepted 12 August 2011

\begin{abstract}
Objective: The Mediterranean diet has been related to a lower risk of some chronic diseases, including cancer. We aim to gain insight into the effects of the main source of fat of this diet on breast cancer, the most common type of malignancy in women.

Design: Data from sixteen experimental series analysing the effects of dietary lipids on mammary carcinogenesis in an animal model, in the context of the international literature on the Mediterranean diet, olive oil and breast cancer risk. Setting: Experimental and human data on the effects of olive oil and Mediterranean diet on breast cancer.

Subjects: An animal model of induced breast cancer and other human and experimental studies in the literature.

Results: Diets rich in extra virgin olive oil (EVOO) exert a negative modulatory effect on experimental breast cancer to a weak promoting effect, much lower than that obtained with a high-corn oil diet. EVOO confers to the mammary adenocarcinomas a clinical behaviour and morphological features compatible with low tumour aggressiveness. This differential effect, in relation to other dietary lipids, may be related to a lower effect on body weight and sexual maturation. In addition, EVOO induced different molecular changes in tumours, such as in the composition of cell membranes, activity of signalling proteins and gene expression. All these modifications could induce lower proliferation, higher apoptosis and lower DNA damage. These results, together with the favourable effect of olive oil reported in the literature when it is consumed in moderate quantities, suggest a beneficial influence of EVOO on breast cancer risk.

Conclusions: Consumption of EVOO in moderate quantities and throughout the lifetime appears to be a healthy choice and may favourably influence breast cancer risk.
\end{abstract}

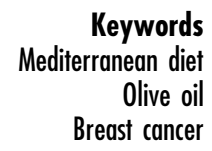

The Mediterranean diet includes a variety of food patterns from different regions of the Mediterranean, and it is characterised by the consumption of an abundance of plant foods (fruit, vegetables, cereals, grains and nuts), dairy products, fish and olive oil as the principal source of fat ${ }^{(1)}$. This diet has been traditionally linked to the longevity of populations from the Mediterranean countries, and it has been associated with a significant improvement in health status, as seen by a reduction in overall mortality, mortality from CVD, incidence of or mortality from cancer, and incidence of other chronic diseases ${ }^{(2)}$. Olive oil, as a major energy source in the Mediterranean diet, is being investigated for its contribution to the reported health benefits of this diet. The main active components of olive oil include monounsaturated lipids (especially oleic acid), phenolic constituents (such as hydroxytyrosol, tyrosol and oleuropein) and squalene ${ }^{(3,4)}$.
Cancer is a major health problem and a leading cause of mortality worldwide. The most commonly diagnosed cancers in men are lung $(16.5 \%$ of the total), prostate $(13 \cdot 8 \%)$ and colorectum $(10 \cdot 0 \%)$, whereas in women they are breast $(22 \cdot 9 \%)$, colorectum $(9 \cdot 4 \%)$ and cervix uteri $(8 \cdot 8 \%)^{(5)}$. Geographical differences in cancer incidence rates indicate a role of environmental factors in the aetiology of this disease, nutrition being one of the most relevant. Epidemiological and especially experimental studies have found a relationship between dietary lipids and some cancers, such as breast and colorectum $^{(6,7)}$. Human data have also shown that women in the Mediterranean European countries have a lower incidence of breast cancer than women in other Western countries $^{(8)}$, and recent prospective studies have associated the Mediterranean diet with a reduction of breast cancer risk ${ }^{(9,10)}$. In fact, it has been proposed that up to 
$25 \%$ of colorectal, $15 \%$ of breast and $10 \%$ of prostate, pancreas and endometrial cancers could be prevented by adhering to this diet ${ }^{(8)}$.

Thus, because strong evidence suggests that consuming the Mediterranean diet may have a protective effect, the aim of the present paper is to analyse the influence of the main source of fat of this diet (olive oil) on breast cancer and the mechanisms involved in this effect.

\section{Effects of extra virgin olive oil on experimental breast cancer: clinical parameters}

The relationship between dietetic factors, especially dietary lipids, and breast cancer has long been analyzsd. Although experimental studies in animals have clearly shown an influence of dietary lipids on breast cancer ${ }^{(7,11-13)}$, heterogeneous human epidemiological results have been reported. While cohort studies have generated conflicting results, case-control analyses and new prospective studies have shown that high fat intake is an important modulator of breast cancer risk ${ }^{(13-16)}$. The main source of fat in the Mediterranean diet is olive oil, but the effects of this oil on breast cancer risk are not well elucidated. Moreover, although dietary habits in the Mediterranean countries have been traditionally linked to the high consumption of olive oil, in recent years the consumption of seed oils rich in $n-6$ PUFA - such as sunflower or corn oils - and other oils has increased. Thus, more scientific evidence is needed in relation to the effects of olive oil and high- $n-6$ PUFA oils on breast cancer. Owing to the difficulty in obtaining data with controlled variables in humans, animal models are widely used to gain insight into the influence of dietetic factors on health. For more than 20 years, we have been using the experimental model of breast cancer chemically induced with 7,12-dimethylbenz $(\alpha)$ anthracene (DMBA) in the female Sprague-Dawley rat ${ }^{(17)}$. The suitability of this model for the possible application of the results in human breast cancer was previously validated, and it is used extensively in breast cancer studies ${ }^{(18,19)}$. For the investigation of the different effects that olive oil and $n-6$ lipids may have on the initiation and promotion of mammary adenocarcinomas, a low-fat diet (with $3 \%$ of corn oil), a high-fat $n-6$ diet (with $20 \%$ of corn oil) and high-fat $n-9$ (with a $17 \%$ of extra virgin olive oil (EVOO) and $3 \%$ of corn oil) diets have been designed and validated ${ }^{(20-22)}$.

To study the effects of dietary lipids on experimental breast cancer, several clinical parameters have been defined, in addition to suitable statistical analysis ${ }^{(23-26)}$. In sixteen experimental series developed in our laboratory, results have always been conclusive: diets with a high content of $n$ - 6 PUFA (corn oil) exert a clear stimulating effect of the experimental mammary carcinogenesis in all the clinical parameters studied ${ }^{(22,27-31)}$ (Fig. 1). This stimulating influence has been mainly observed in the promotion stage of carcinogenesis, although an influence
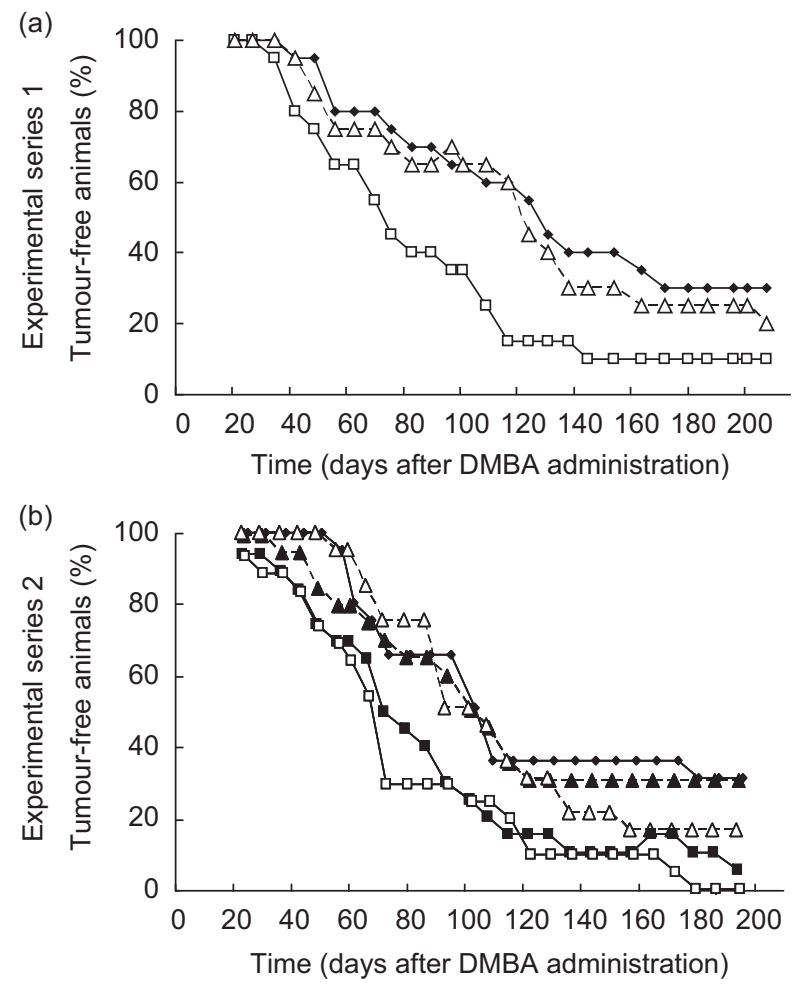

Fig. 1 Effect of an HEVOO diet and an HCO diet on body weight and mass of female rats at 235-254 d of age; (a) body weight (g); (b) BMI $\left(\mathrm{g} / \mathrm{cm}^{2}\right)$. HEVOO, high-extra virgin olive oil group; HCO, high-corn oil group; LF, low-fat group. Values are mean and SE. ${ }^{*} P<0.05$ compared to control LF group (Tukey's test; $n$ 20/group)

on the initiation stage cannot be ruled out. In contrast, the diet high in EVOO, with the same total content in fat as the high-corn oil (HCO) diet, has noticeably shown a different effect (Fig. 1). Thus, depending on the parameter studied, the high-olive oil diet exerted a weak stimulatory effect to a protective effect on mammary carcinogenesis. In all the experimental studies developed in our laboratory, the high-EVOO diet, in comparison with the HCO diet, consistently increased tumour latency (retarding the appearance of tumours) and decreased tumour incidence, multiplicity and volume ${ }^{(7,22,30-33)}$. Moreover, in some of the experimental series, we have observed that the olive oil diet reduced tumour content and volume, even in comparison with a control low-fat diet, which suggested a protective effect of the olive oil on mammary carcinogenesis ${ }^{(22,33)}$. The study of tumour regression, as a proposed protective effect of this higholive oil diet, showed a slow progression of tumours in the animals fed that diet, rather than a real regression ${ }^{(22)}$. The differences observed in the experimental series can be related to the different timing of the dietary intervention, as well as to distinct varieties of oil used, as it has been reported that the influence of olive oil on carcinogenesis depends on the proportions of oleic acid and minor components of the oil ${ }^{(34)}$. Such findings are of 
interest considering the high percentage of olive oil used in this experimental diet, as it should be taken into consideration that all high-fat diets may have an unspecific stimulatory effect on carcinogenesis ${ }^{(35)}$. In this sense, a positive association between the dietary energy supply and cancer mortality rates has been shown, and energy restriction has an indiscriminate inhibitory effect on carcinogenesis $^{(36)}$. In the literature, experimental studies on the effects of olive oil in the diet have reported inconsistent results $^{(7)}$. Administration of diets with a lower percentage of oil (7\% and 15\%) during prenatal life has significantly increased the number of tumour-free rats ${ }^{(37)}$.

\section{Effects of extra virgin olive oil on experimental breast cancer: histopathological characteristics}

Our group has also characterised morphologically, for the first time according to our knowledge, the effect that dietary lipids may exert on experimental breast cancer. Previously, a histological grading system adapted to rat mammary carcinomas was developed ${ }^{(38,39)}$. These studies first showed the correspondence between the histological pattern and the clinical characteristics of each tumour, finding a higher degree of biological aggressiveness in tumours with higher histopathological degrees, desmoplastic reaction, limfoplasmocitic infiltrate, tumour necrosis and prevalent cribiform architectural patterns ${ }^{(38,39)}$. Furthermore, the analysis of the effects of different diets showed that the HCO diet promoted adenocarcinomas with a high histological degree, stromal invasion, more prominent tumour necrosis and a frequent cribiform pattern in comparison with the controls and high-olive oil diet. In contrast, animals fed a diet rich in EVOO developed adenocarcinomas with a low histopathological grade, few invasive and necrotic areas (similar to the tumours from the control group) and extensive papillary areas ${ }^{(40)}$. In conclusion, tumours from the animals fed the high-EVOO diet clearly showed a lower degree of tumour malignancy than those from the animals fed the HCO diet, being more similar to control tumours. All these results are compatible with the lower degree of clinical malignancy and with the non-promoting effect of a diet rich in $\mathrm{EVOO}^{(40)}$.

\section{Mechanisms of action of extra virgin olive oil on breast cancer: growth and sexual maturation}

The mammary gland has the particularity that, unlike other organs, after birth it remains highly undifferentiated until the onset of puberty. Reproductive events increasing the number of menstrual cycles and therefore lifetime exposure to oestrogens, such as early menarche, have been associated with increased risk of breast cancer ${ }^{(6)}$. Thus, modifications in growth and sexual maturation due to the effect of nutrition may modify the susceptibility to
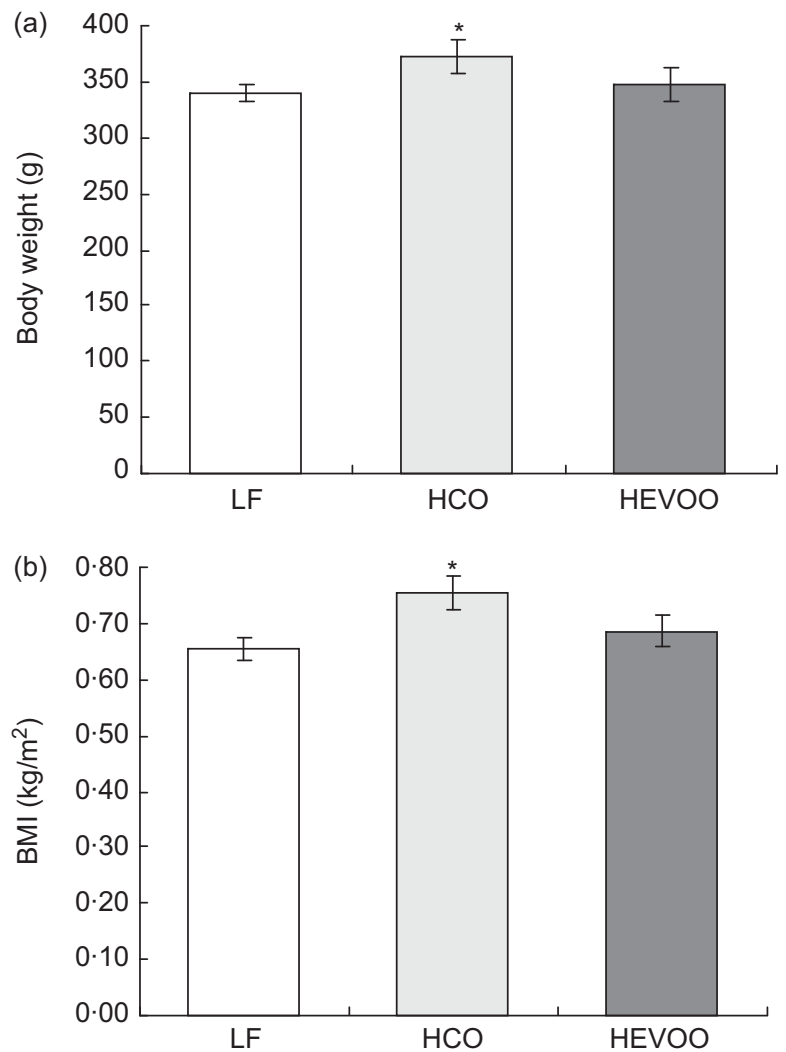

Fig. 2 Effects of an HEVOO diet and an HCO diet on rat DMBA-induced mammary carcinogenesis in two different experimental series. Tumour-free animals: percentage of rats without palpable tumours. Experimental series 1: animals were fed a low-fat diet (3\% corn oil; w/w), an HCO diet $(20 \%$ corn oil) or an HEVOO (3\% corn oil $+17 \%$ olive oil) diet after the induction with the carcinogen (promotion phase of carcinogenesis $(P)$ ). Experimental series 2: animals were fed the low fat, $\mathrm{HCO}$ or HEVOO diet from weaning (initiation and promotion phase of the carcinogenesis (IP)) or after the induction with the carcinogen (only promotion phase $\mathrm{P}$ ). HEVOO, highextra virgin olive oil group; HCO, high-corn oil group; DMBA, 7,12-dimethylbenz $(\alpha)$ anthracene; LF, groups fed the low-fat diet; $\mathrm{HCO}_{\mathrm{IP}}$, group fed the $\mathrm{HCO}$ diet from weaning (initiation + promotion); $\mathrm{HCO}_{\mathrm{P}}$, groups fed the $\mathrm{HCO}$ diet from induction (promotion); HEVOO ${ }_{\mathrm{IP}}$, group fed the HEVOO diet from weaning (initiation + promotion); $\mathrm{HEVOO}_{\mathrm{P}}$, groups fed the HEVOO diet from induction (promotion)

mammary transformation, advancing, retarding or expanding the windows of this susceptibility.

We observed that a diet high in corn oil increased the body weight of the rats as well as the BMI. In contrast, the high-olive oil diet, with the same percentage of fat as the HCO diet, did not modify body weight or mass in relation to the low-fat diet ${ }^{\text {(31) }}$ (Fig. 2). Several studies have also reported an effect of the high- $n-6$ PUFA diet on body weight, but there are few experimental data regarding the effects of the olive oil diet. In this sense, a $30 \%$ EVOO diet has also been shown to produce lower bodyweight gain in rats when compared to a $30 \%$ corn oil diet ${ }^{(41)}$. On the other hand, there are human epidemiological data suggesting 
Table 1 Effect of an LF, HCO and an HEVOO diet on day of arrival at different states of maturation in female rats

\begin{tabular}{|c|c|c|c|c|c|c|c|c|c|}
\hline & \multicolumn{3}{|c|}{$\mathrm{LF}(n 81)$} & \multicolumn{3}{|c|}{$\mathrm{HCO}(n 31)$} & \multicolumn{3}{|c|}{ HEVOO ( $n$ 31) } \\
\hline & Mean & SE & Median & Mean & SE & Median & Mean & SE & Median \\
\hline \multicolumn{10}{|l|}{ Day of arrival at: } \\
\hline Maturation state 1 & $34 \cdot 8$ & $0 \cdot 31$ & 34 & $32 \cdot 2^{*}$ & $0 \cdot 36$ & $32^{*}+$ & $32 \cdot 7^{*}$ & 0.33 & 34 \\
\hline Maturation state 2 & $38 \cdot 1$ & $0 \cdot 21$ & 38 & $36 \cdot 6^{*}$ & 0.23 & $36^{*}$ & $36 \cdot 5^{\star}$ & 0.23 & $36^{*}$ \\
\hline Maturation state 3 & $44 \cdot 1$ & $0 \cdot 33$ & 44 & $42 \cdot 2^{*}$ & 0.47 & $42^{*}$ & $42 \cdot 8$ & 0.54 & 43 \\
\hline
\end{tabular}

LF, low fat; HCO, high-corn oil; HEVOO, high-extra virgin olive oil.

${ }^{\star} P<0.05$ compared to control LF group.

$+P<0.05$ compared to HEVOO group (parametric Tukey's test for mean, non-parametric Mann-Withnney's $U$ test for median).

that the Mediterranean diet may have a protective effect on obesity ${ }^{(42-45)}$.

Sexual maturation has also been studied in rats fed these high-fat diets. Although a high amount of fat in the diet generally advances the onset of puberty, we observed different effects depending on the type of oil consumed. The onset of puberty in animals fed the HCO diet was significantly advanced, followed by animals fed a high-EVOO diet, and the control animals ${ }^{(31)}$ (Table 1). No differences were found either in body weight or mass when arriving at each stage, thus suggesting that the reproductive morphological changes are related to the acquisition of a threshold level of body mass, as has been already reported in humans ${ }^{(46)}$. This threshold weight would appear earlier in the animals of the HCO group, followed by the high-EVOO group and the controls. These results were in accordance with the study of a marker of puberty, the hypothalamic expression of kisspeptin ${ }^{(47)}$. The mRNA levels of kisspeptin in the hypothalamus of rats fed the HCO diet suggested higher expression of this marker at around puberty, while no differences were observed in the animals fed the EVOO diet in relation to the controls. No differences were observed in other parameters of maturation due to the effect of diets (uterine weight, time span among stages, time from vaginal opening to first oestrus or cycle), which also suggested that high-fat diets may advance the process of maturation but did not change its evolution ${ }^{(31)}$.

\section{Mechanisms of action of extra virgin olive oil on breast cancer: hormonal status}

Breast cancer is an oestrogen-dependent neoplasia, and thus may be influenced by modifications in hormonal status $^{(6)}$. Thus, the possible effect of dietary lipids on serum concentrations of luteinizing hormone, folliclestimulating hormone, oestradiol, progesterone, prolactin, insulin and corticosterone has been analysed, but no significant modifications due to the effect of high-olive oil or HCO diets ${ }^{(48)}$ (E Escrich, unpublished results) have been found. In the literature, there are discordant results regarding the influence of dietary lipids on hormone levels. For example, pregnant rats fed with a high- $n-6$
PUFA diet have been shown to either increase ${ }^{(49)}$ or not modify ${ }^{(50)}$ serum oestradiol levels. Other authors have found lower concentrations of oestradiol in lactating dams fed a $7 \%$ olive oil diet in comparison to those fed a $7 \%$ corn oil diet ${ }^{(51)}$. The discrepancy among results may be related to the cycling nature of hormones, as their serum concentrations are highly dependent on the hour of the day and the phase of the oestrous cycle ${ }^{(52)}$.

To better understand the effects that dietary lipids may have on hormonal status, we also analysed the expression levels of hormone receptors in the mammary gland and in the experimental tumours, finding little difference due to the effect of the high-fat diets. Thus, analyses of the expression of estrogen receptors (ER $\alpha, \mathrm{ER} \beta 1$ and ER $\beta 2)$ showed no significant differences due to the effect of diets. We observed higher levels of progesterone receptors in the mammary gland of rats fed the EVOO diet in relation to the animals fed the HCO diet at the age around puberty, which may be related to the development of lobulo-alveolar structures $^{(31)}$. The few changes observed suggest that the modulation of the expression levels of these hormone receptors are not a major mechanism by which these highfat diets may influence mammary gland biology.

\section{Mechanisms of action of extra virgin olive oil on breast cancer: morphology and differentiation of the mammary gland}

The mammary gland is a target of sexual maturity. Various studies on animals have shown that the risk of malignant transformation of this tissue can be highly dependent on the influence of early life events, and highlight the importance of the degree of differentiation at the time of a carcinogenic insult ${ }^{(53)}$. Thus, we characterised the differentiation degree of the mammary gland by quantifying the different epithelial structures in the periphery of the gland. Other authors have suggested that high-fat diets, such as HCO diet, may influence mammary carcinogenesis by increasing the number of the epithelial structures that are target of the carcinogen (the undifferentiated terminal end buds (TEB) $)^{(49,53)}$. However, we found little influence from either of the high-fat diets on the number of TEB, terminal buds, alveolar buds or lobular structures of the breast tissue ${ }^{(31)}$. 
To further characterise the degree of differentiation of the mammary gland, mRNA expression of $\beta$-casein, classically considered a molecular differentiation marker of this gland, has also been analysed. $\beta$-Casein expression increased in the mammary tissue over time, but no significant differences were found due to the effect of dietary lipids. Although $\beta$-casein mRNA levels inversely correlated with the number of undifferentiated terminal ducts, we did not find a correlation with TEB, the main target of carcinogens ${ }^{(53)}$, or, therefore, with the susceptibility of the mammary gland to transformation.

\section{Mechanisms of action of extra virgin olive oil on breast cancer: molecular changes in tumours}

The molecular mechanisms by which dietary lipids may exert their effects on the development of mammary cancer are not well understood. Experimental studies support the hypothesis of the existence of a specific effect aside from the unspecific one derived from the energy supply ${ }^{(35)}$. Some of the proposed mechanisms, to which we have contributed with experimental data, include modifications of the structure and function of cell membranes, modulation of gene expression or influence on cell signalling pathways ${ }^{(54)}$. Moreover, several studies have reported beneficial effects of olive oil through other mechanisms, such as modifications in oxidative stress and changes in immune system function ${ }^{(7,54,55)}$. Lipids could act through several of these and other mechanisms in an integrated, simultaneous and/or sequential way.

\section{Modifications of cell membranes}

Lipids are an essential component of cell membranes and thus regulate their biological activity. Membrane lipid composition may be altered by dietary lipids, mainly by $n-3$ PUFA and the $n-3 / n-6$ ratio of the $\operatorname{diet}^{(56)}$. Few studies have been carried out regarding the influence of olive oil on membrane composition, but there is evidence that adherence to the Mediterranean diet may affect the structural properties of the erythrocyte cell membrane of hypertensive patients. This influence has been related to the beneficial effect of the Mediterranean diet on hypertension ${ }^{(57)}$. A high content of polyunsaturated lipids in membranes increases the fluidity and the susceptibility to peroxidation ${ }^{(56)}$. We have studied the membrane composition of experimental tumours by analysing fourteen different fatty acids in six lipidic fractions. A high- $n-6$ PUFA diet changed the tumour lipid profile, increasing the $18: 2 n-6$ relative content and decreasing that of the 18:1n-9 (significantly in three lipidic fractions: phosphatidylcholine, free fatty acids and TAG) ${ }^{(28)}$. Taking into consideration that those tumours had more aggressive clinical and histopathological behaviour, these results would be in accordance with the lower effect of MUFA on membrane fluidity and the lower susceptibility of these lipids to peroxidation, thus decreasing the oxidative damage in membranes.

\section{Effects on gene expression}

It has long been known that different dietary components can modulate the expression of specific genes. Although data regarding the effect of dietary lipids in genes with a role in cancer are scarce, there is a wealth of information reporting the effects of such dietary compounds on genes of the metabolism ${ }^{(58)}$. Moreover, the effects of dietary lipids could be modified by the disease, as we have observed changes in the normal regulation of the expression of CPT-I, HMGCoA synthase and PPAR $\alpha$ by high-fat diets in the liver of tumour-bearing rats ${ }^{(59)}$.

The healthy benefits of the Mediterranean diet have been partly associated with changes in the expression of atherosclerosis-related genes through olive oil polyphenols ${ }^{(60)}$. Thus, olive oil may exert some of its influence on breast cancer through the modulation of expression of genes involved in the carcinogenesis process. We found in experimental mammary tumours that the high-EVOO diet and the HCO diet had different influences on the modulation of the expression of the ErbB family of membrane receptors, especially c-erbB1. Hence, the HCO diet increased the ratio between the $9.5 \mathrm{~kb}$ mRNA of EGFR (coding the functional full-length receptor) and the $2.7 \mathrm{~kb}$ mRNA (coding an inactive truncated receptor), whereas the high-EVOO diet decreased this ratio in the mammary tumours $^{(30)}$. On the other hand, the expression levels of p21Ras, a key transductor of ErbB proliferative signalling that is frequently involved in human mammary carcinogenesis $^{(61)}$, were not modified by these dietary lipids ${ }^{(29)}$. High-fat diets did not modify either the expression levels of HMG-CoA reductase or squalene synthase, two genes that codify enzymes of the mevalonate pathway ${ }^{(62)}$. Mevalonate is the source of the prenyl groups needed for the posttranslational modification of p21Ras and its subsequent activation $^{(63)}$.

Cell dedifferentiation is part of the alterations linked to cancer, and thus our group studied whether a high-EVOO diet and a HCO diet changed the degree of molecular differentiation of DMBA-induced mammary tumours ${ }^{(64)}$. We analysed the expression of known mammary differentiation markers, $\alpha$-casein, $\beta$-casein and transferrin, in addition to $\beta$-actin and its transporter protein ZBP1. In the mammary adenocarcinomas, the expression levels of $\alpha$-casein, $\beta$-casein and transferrin were not related to the degree of morphological differentiation or to the clinical behaviour of the tumours, which suggested that these genes were not good biomarkers of the modifications that the experimental diets conferred to the adenocarcinomas. The HCO diet, but not the high-EVOO diet, increased the $\beta$-actin mRNA levels but not those of the protein. This last result, in addition to the increase observed in the transporter ZBP1 as a result of the HCO diet, suggested a deregulated transport and translation of $\beta$-actin that was 


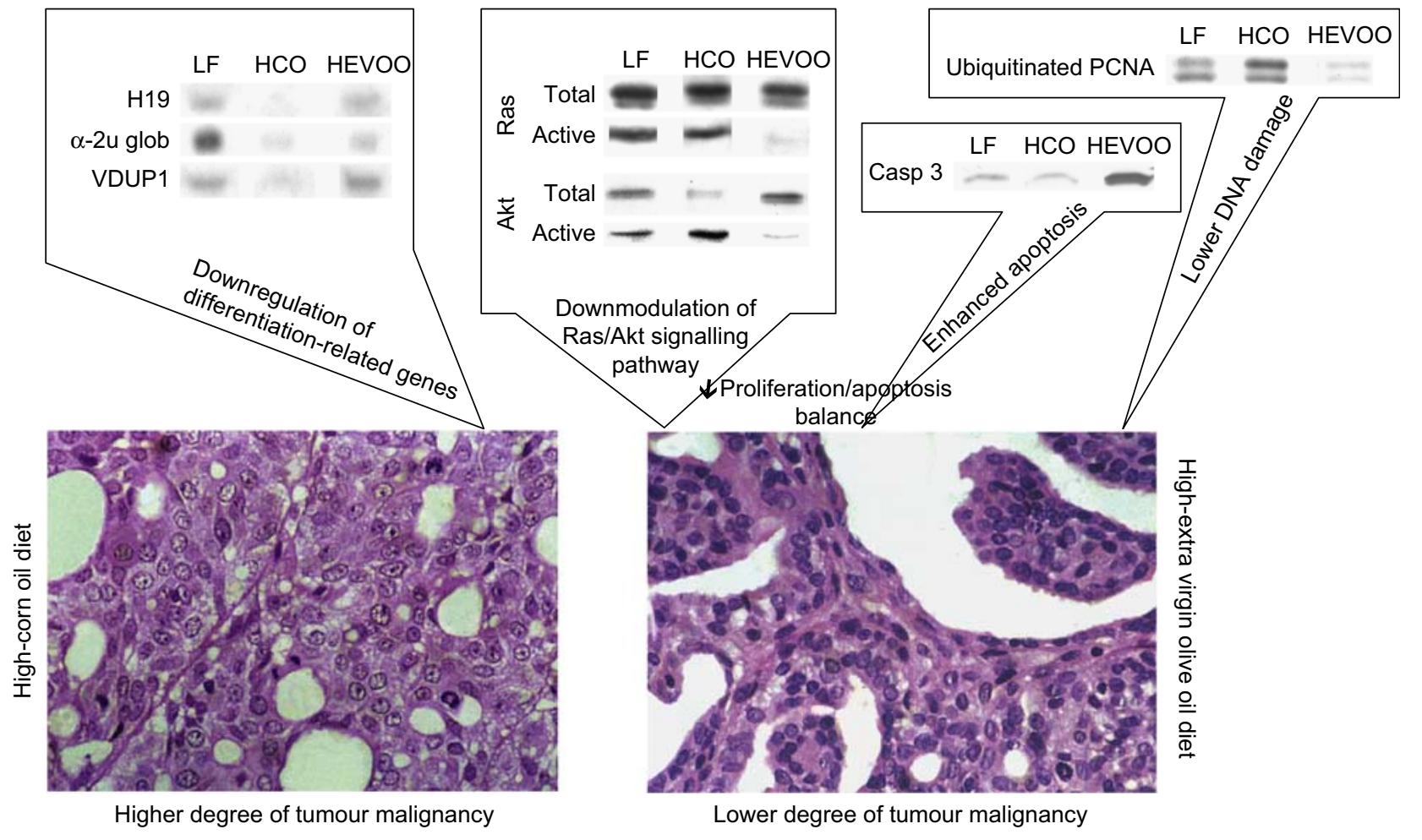

Fig. 3 (colour online) Molecular mechanisms of the effects of an HEVOO diet and an HCO diet on DMBA-induced tumour malignancy. Animals fed the HCO diet displayed tumours with a higher histopathological degree of tumour aggressiveness, while animals fed the HEVOO diet displayed tumours similar to those of the animals fed a low-fat diet (LF). The HCO diet downregulated genes with a role in cell differentiation. The HEVOO diet downmodulated the Ras/Akt signalling pathway, which may elicit lower proliferation and higher apoptosis. Moreover, caspase 3 (casp 3) was upregulated in tumours from animals fed the HEVOO, thus suggesting a proliferation-apoptosis balance shifted to apoptosis. Furthermore, the tumours from the HEVOO group showed lower levels of ubiquitinated PCNA, which is related to DNA damage, suggesting lower levels of this damage due to the effect of the EVOO diet (HEVOO, high-extra virgin olive oil group; HCO, high-corn oil group; DMBA, 7,12-dimethylbenz( $\alpha)$ anthracene; proliferating cell nuclear antigen (PCNA))

associated with the most malignant phenotype of the tumours from animals fed a diet rich in corn oil, but not with those of the animals fed the high-EVOO diet ${ }^{(64)}$.

New screening technologies allowed different experimental approaches in order to gain insight into the modulation of gene expression by dietary lipids. cDNA microarrays analyses were used to discover four genes significantly downregulated due to the effect of the HCO diet in tumours ${ }^{(65)}$ but not modified by the high-EVOO diet $^{(66)}$. The genes differentially modulated in the adenocarcinomas of animals fed a diet rich in n-6 PUFA were a2u-globulin, VDUP1, the imprinted gene $H 19$ and an unknown gene (Fig. 3). Experimental evidence suggests that these known genes are potentially related to differentiation and cell proliferation, thus being involved in the tumour-stimulating effect of the HCO diet ${ }^{(65)}$. Moreover, the IGFII gene, related to proliferation and reciprocally imprinted with H19, was upregulated by the HCO diet and downregulated by the high-EVOO diet. Furthermore, both high-fat diets had an opposing effect on the activity of thioredoxin, an oxidoreductase inhibited by VDUP1 that promotes cell growth and has an antiapoptotic action. This result was in accordance with the opposing effect of the HCO and EVOO diets on mammary carcinogenesis $^{(65,66)}$.

\section{Influence on signalling patbways}

Our investigations have also focused on the effects that olive oil diet may have on signalling pathways with an important role in mammary development and human breast cancer, such as the ErbB-Ras and their main effectors (Erk1/2, PI3K/Akt, RalGDS/Ral) ${ }^{(61)}$. The protein levels and activity of the ErbB1, ErbB2 and ErbB3 were not altered due to the HCO diet or the high-olive oil diet, but we observed a significantly decreased expression in the $80 \mathrm{kDa}$ ErbB4-truncated protein due to the effect of the high-EVOO $\operatorname{diet}^{(62)}$.

We next investigated the effect of the olive oil diet on p21Ras expression and activity. Animals fed this diet developed tumours with significantly higher levels of p21Ras protein, but with a significantly decreased activity (Fig. 3). In contrast, no changes were observed in p21Ras function as a result of the HCO diet, suggesting that p21Ras may have a role in the negative modulatory effect 
of olive oil on breast cancer, whereas it is not involved in the stimulatory effect of the HCO diet ${ }^{(62)}$. Several mechanisms of action for this decreased activity have been addressed: the tumour mutation status of Ha-ras1 and the expression of two key enzymes of the mevalonate pathway, the HMG-CoA reductase and squalene synthase (SQS). The experimental diets did not modify the rate of the activating point mutation in the codon 61 of the gene c-Ha-ras1, or the mRNA expression levels of HMG-COA and SQS in the mammary adenocarcinomas (as mentioned above). Other experimental studies have suggested that an olive oil diet may have a protective effect on colon cancer through the effect of squalene, a minor compound of olive oil, inhibiting the HMG-CoA reductase ${ }^{(67)}$. However, such effects have been observed at very high concentrations of squalene ( $1 \%$ of diet), and our results did not support the hypothesis of a chemopreventive effect of olive oil diet through squalene on HMG-CoA reductase inhibition, at least at the expression level ${ }^{(62)}$.

The analysis of the main effectors of p21Ras showed increased ERK1/2 activity and reduced ERK1/2 expression due to the effect of both high-fat diets. These results suggested an unspecific effect of such diets upregulating the ERK1/2 proliferating pathway, which would not be elicited by ErbB receptors and could be mediated, at least partly, by a p21Ras-independent mechanism. No differences were found in the expression or activity of RalA and RalB proteins due to the effect of the experimental diets. On the other hand, the EVOO diet decreased both Akt expression and activation, whereas the HCO diet only decreased Akt protein levels ${ }^{(62)}$ (Fig. 3). A reduced activity of Akt has been associated with induction of apoptosis ${ }^{(68)}$; thus, these results suggested that the higholive oil diet may downregulate the pro-survival p21/Akt pathway in the mammary tumours. Few data have been published regarding the effects of olive oil on these pathways, but it has been shown that in breast cancer cells oleic acid both increased ${ }^{(69)}$ and decreased ${ }^{(70)}$ active Akt, whereas hydroxytirosol abolished Akt phosphorylation and induced transient Erk $1 / 2$ phosphorylationdephosphorylation in colon cancer cells ${ }^{(71)}$.

The influence of the high-olive oil diet on mammary tumours may thus result in upregulation of ERK1/2 pathway concomitantly with a downregulation in Akt signalling. There are experimental data in the literature suggesting that this relative balance between ERK1/2 and Akt pathways may result in pro-apoptotic signalling ${ }^{(72,73)}$. To address the hypothesis that the EVOO diet induced cell apoptosis, we analysed the levels of the activated caspase-3, which is considered the main executioner of the caspase cascade involved in both extrinsic and intrinsic apoptosis ${ }^{(74)}$. The tumours from the animals fed the olive oil diet had significantly higher levels of this protein, which was concordant with the proposed proapoptotic effect of the olive oil ${ }^{(62)}$ (Fig. 3). These results are in line with other studies that have reported that olive oil, oleic acid and minor compounds of olive oil can modulate apoptosis ${ }^{(51,75-77)}$.

Apoptosis is a crucial process in cancer development, and it has been suggested that tumour growth is partly determined by the balance between cell proliferation and apoptosis. The histopathological analysis of the mitotic activity and the protein expression levels of the mitotic marker proliferating cell nuclear antigen (PCNA) indicated that the high-EVOO diet did not exert a significant effect on tumour cell proliferation, in contrast to the HCO diet, which significantly increased tumour mitotic activity ${ }^{(40,62)}$. This last result was consistent with an influence of HCO diet on the survival and proliferation signalling pathways and with its stimulatory effect on mammary carcinogenesis $^{(7,22,27-33)}$. Moreover, the high-EVOO diet decreased the levels of monoubiquitylated PCNA, which is associated with DNA damage, in the mammary tumours $^{(62)}$ (Fig. 3). The effect that olive oil may have on the proliferation of tumour cells is still not well elucidated. In vitro experiments have found evidence that some minor compounds of olive oil, such as hydroxytyrosol and oleuropein, inhibit proliferation of breast cancer cells ${ }^{(75)}$, while oleate has been reported to have a stimulatory effect on cell proliferation ${ }^{(76)}$.

\section{Conclusions}

There is increasing epidemiological and experimental evidence indicating the beneficial effect of the Mediterranean diet, and in particular of EVOO, on the progression of some cancers, especially breast cancer. This effect could be the result of its MUFA (oleic acid) and its minor compounds.

In an experimental model of mammary cancer, we have observed that a high-EVOO diet clearly has a different effect on breast carcinogenesis if compared with the effect of other diets (an $n$-6 PUFA enriched diet) with the same amount of fat, showing the importance of the type of oil consumed beyond its energy content. Some of the parameters that we have studied in relation to the clinical and histopathological behaviour of tumours, in addition to experimental data published in the literature using lower percentages of olive oil in the diet, suggest that EVOO may have a beneficial effect on breast cancer risk if its consumption is moderated. The mechanisms by which this type of oil may exert its effects are diverse, such as a protective effect on obesity or molecular influences eliciting a balance between proliferation and apoptosis shifted in favour of apoptosis or lower levels of DNA damage in tumours. These data may contribute to the published evidence of the healthy effect of the Mediterranean diet and highlight that the use of EVOO as the source of fat from childhood may be considered as a healthy choice. 


\section{Acknowledgements}

The present study was supported by grants from the 'Plan Nacional de I+D+I 2004-2007' (AGL2006-07691/ALI); 'Fundación Patrimonio Comunal Olivarero 2008-2012' (FPCO2008-165-396); 'Agencia para el Aceite de Oliva del Ministerio de Medio Ambiente y de Medio Rural y Marino 2008-2012' (AAO2008-165·471); 'Organización Interprofesional del Aceite de Oliva Español 2009-2013' (OIP2009-165.646) and the 'Departaments d'Agricultura, Alimentació i Acció Rural, i de Salut de la Generalitat de Catalunya' (GC2010-165•000). The sponsors had no role in the study design, data collection and analysis, interpretation of results, the preparation of the manuscript, the decision to submit the manuscript for publication or the writing of the manuscript. E.E. conceived the study, its design, and coordinated the project. All authors are involved with the study design and review of the literature, data analysis and interpretation, made critical revisions of the paper, and provided approval for its publication. The authors thank Raquel Escrich and Irmgard Costa for their collaboration on these studies.

\section{References}

1. Willett WC, Sacks F, Trichopoulou A et al. (1995) Mediterranean diet pyramid: a cultural model for healthy eating. Am J Clin Nutr 61, 6 Suppl., 1402S-1406S.

2. Sofi F, Cesari F, Abbate R et al. (2008) Adherence to Mediterranean diet and health status: meta-analysis. BMJ 337, a1344.

3. Hashim YZ, Eng M, Gill CI et al. (2005) Components of olive oil and chemoprevention of colorectal cancer. Nutr Rev 63, 374-386.

4. Owen RW, Giacosa A, Hull WE et al. (2000) Olive-oil consumption and health: the possible role of antioxidants. Lancet Oncol 1, 107-112.

5. Ferlay J, Shin HR, Bray F et al. (2010) Estimates of worldwide burden of cancer in 2008: GLOBOCAN 2008. Int J Cancer 127, 2893-2917.

6. World Cancer Research Fund/American Institute for Cancer Research (2007) Food, Nutrition, Physical Activity, and the Prevention of Cancer: A Global Perspective. Washington, DC: AICR.

7. Escrich E, Solanas M \& Moral R (2006) Olive oil, and other dietary lipids, in cancer: experimental approaches. In Olive Oil and Health, pp. 317-374 [JL Quiles, MC RamírezTortosa and P Yaqoob, editors]. Oxford: CABI Publishing.

8. Trichopoulou A, Lagiou P, Kuper H et al. (2000) Cancer and Mediterranean dietary traditions. Cancer Epidemiol Biomarkers Prev 9, 869-873.

9. Cottet V, Touvier M, Fournier A et al. (2009) Postmenopausal breast cancer risk and dietary patterns in the E3N-EPIC prospective cohort study. Am J Epidemiol 170, $1257-1267$.

10. Trichopoulou A, Bamia C, Lagiou P et al. (2010) Conformity to traditional Mediterranean diet and breast cancer risk in the Greek EPIC (European Prospective Investigation into Cancer and Nutrition) cohort. Am J Clin Nutr 92, 620-625.

11. Wynder EL, Cohen LA, Muscat JE et al. (1997) Breast cancer: weighing the evidence for a promoting role of dietary fat. J Natl Cancer Inst 89, 766-775.
12. Fay MP, Freedman LS, Clifford CK et al. (1997) Effect of different types and amounts of fat on the development of mammary tumors in rodents: a review. Cancer Res 57, 3979-3988.

13. Bartsch H, Nair J \& Owen RW (1999) Dietary polyunsaturated fatty acids and cancers of the breast and colorectum: emerging evidence for their role as risk modifiers. Carcinogenesis 20, 2209-2218.

14. Smith-Warner SA, Spiegelman D, Adami HO et al. (2001) Types of dietary fat and breast cancer: a pooled analysis of cohort studies. Int J Cancer 92, 767-774.

15. Howe GR, Hirohata T, Hislop TG et al. (1990) Dietary factors and risk of breast cancer: combined analysis of 12 case-control studies. J Natl Cancer Inst 82, 561-569.

16. Schulz M, Hoffmann K, Weikert C et al. (2008) Identification of a dietary pattern characterized by high-fat food choices associated with increased risk of breast cancer: the European Prospective Investigation into Cancer and Nutrition (EPIC)-Potsdam Study. Br J Nutr 100, 942-946.

17. Escrich E (1987) Mammary cancer model induced by dimethylbenz(a)anthracene: a good experimental tool for the study of tumour markers. Int J Biol Markers 2, 109-119.

18. Escrich E (1987) Validity of the DMBA-induced mammary cancer model for the study of human breast cancer. Int $J$ Biol Markers 2, 197-206.

19. Russo J, Gusterson BA, Rogers AE et al. (1990) Comparative study of human and rat mammary tumourigenesis. Lab Invest 62, 244-278.

20. Escrich E, Solanas M \& Segura R (1994) Experimental diets for the study of lipid influence on induced mammary carcinoma in rats: I - diet definition. In Vivo $\mathbf{8}$, 1099-1106.

21. Escrich E, Solanas M, Ruiz de Villa MC et al. (1994) Experimental diets for the study of lipid influence on induced mammary carcinoma in rats: II - suitability of the diets. In Vivo 8, 1107-1112.

22. Solanas M, Hurtado A, Costa I et al. (2002) Effects of a high olive oil diet on the clinical behavior and histopathological features of rat DMBA-induced mammary tumors compared with a high corn oil diet. Int J Oncol 21, 745-753.

23. Escrich E, Ribalta T, Muntané J et al. (1991) Effects of an androgenic derivative on pre-established mammary tumours chemically induced in the rat. $J$ Cancer Res Clin Oncol 117, 575-582.

24. Ruiz de Villa MC, Cabral MSE, Escrich E et al. (1999) A non parametric regression approach to repeated measures analysis in cancer experimental. J Appl Stat 26, 601-611.

25. El Halimi R, Ocaña J, Ruiz de Villa MC et al. (2003) Modelling experimental mammary adenocarcinoma growth, using nonlinear mixed-effects models and S-Plus. InterStat, http://jscs.stat.vt.edu/interstat/articles/2003/abstracts/ 0309002.html-ssi

26. Goncalves MH, Cabral MS, Ruiz de Villa MC et al. (2007) Likelihood approach for count data in longitudinal experiments. Comput Stat Data Anal 51, 6511-6520.

27. Solanas M, Moral R \& Escrich E (2000) Effects of dietary (n-6) polyunsaturated lipids on experimental mammary carcinogenesis. J Women's Cancer 2, 67-72.

28. Escrich E, Solanas M, Soler M et al. (2001) Dietary polyunsaturated n-6 lipids effects on the growth and fatty acid composition of rat mammary tumors. J Nutr Biochem 12, 536-549.

29. Solanas M, Moral R \& Escrich E (2001) The stimulating effect of a high-fat n-6 polyunsaturated diet on rat DMBAinduced mammary tumors is not related to changes in c-Ha-ras1 mRNA tumor expression. Nutr Res 21, 1261-1273.

30. Moral R, Solanas M, García G et al. (2003) Modulation of EGFR and neu expression by $n-6$ and $n-9$ high-fat diets in experimental mammary adenocarcinomas. Oncol Rep 10, 1417-1424. 
31. Moral R, Escrich R, Solanas M et al. (2011) Diets high in corn oil or extra-virgin olive oil provided from weaning advance sexual maturation and differentially modify susceptibility to mammary carcinogenesis in female rats. Nutr Cancer 63, 410-420.

32. Escrich E, Ramírez-Tortosa MC, Sanchez-Rovira $\mathrm{P}$ et al (2006) Olive oil in the prevention and the progression of cancer. Nutr Rev 64, 40-52.

33. Escrich E, Solanas M, Moral R et al. (2006) Are the olive oil and other dietary lipids related to cancer? Experimental evidence. Clin Transl Oncol 8, 868-883.

34. Cohen LA, Epstein M, Pittman B et al. (2000) The influence of different varieties of olive oil on $\mathrm{N}$-methylnitrosourea (NMU)-induced mammary tumorigenesis. Anticancer Res 20, 2307-2312.

35. Guthrie N \& Carroll KK (1999) Specific versus non-specific effects of dietary fat on carcinogenesis. Prog Lipid Res $\mathbf{3 8}$, 261-271.

36. Kritchevsky D (1999) Caloric restriction and experimental carcinogenesis. Toxicol Sci 52, 13-16.

37. Kossoy G, Stark A, Tendler Y et al. (2002) Transplacental effects of high fat diets on functional activity of the spleen and lymph nodes, cell kinetics and apoptosis in mammary gland tumors in female rat offspring. Int J Mol Med 10, 773-778.

38. Costa I, Esquius J, Solanas M et al. (2001) Histopathologic characteristics of chemically induced mammary adenocarcinomas in rats fed diets high in (n-6) polyunsaturad lipids. Virchows Archiv 439, 310-311.

39. Costa I, Solanas M \& Escrich E (2002) Histopathologic characterization of mammary neoplastic lesions induced with 7,12 dimethylbenz(alpha)anthracene in the rat: a comparative analysis with human breast tumors. Arch Pathol Lab Med 126, 915-927.

40. Costa I, Moral R, Solanas M et al. (2004) High-fat corn oil diet promotes the development of high histologic grade rat DMBA-induced mammary adenocarcinomas, while high olive oil diet does not. Breast Cancer Res Treat $\mathbf{8 6}$, 225-235.

41. Oi-Kano Y, Kawada T, Watanabe T et al. (2007) Extra virgin olive oil increases uncoupling protein 1 content in brown adipose tissue and enhances noradrenaline and adrenaline secretions in rats. $J$ Nutr Biochem 18, 685-692.

42. Buckland G, Bach A \& Serra-Majem L (2008) Obesity and the Mediterranean diet: a systematic review of observational and intervention studies. Obes Rev 9, 582-593.

43. Soriguer F, Almaraz MC, Ruiz-de-Adana MS et al. (2009) Incidence of obesity is lower in persons who consume olive oil. Eur J Clin Nutr 63, 1371-1374.

44. Romaguera D, Norat T, Vergnaud AC et al. (2010) Mediterranean dietary patterns and prospective weight change in participants of the EPIC-PANACEA project. $A m J$ Clin Nutr 92, 912-921.

45. Perez-Lopez FR, Chedraui P, Haya J et al. (2009) Effects of the Mediterranean diet on longevity and age-related morbid conditions. Maturitas 64, 67-79.

46. Vizmanos B \& Martí-Henneberg C (2000) Puberty begins with a characteristic subcutaneous body fat mass in each sex. Eur J Clin Nutr 54, 203-208.

47. Castellano JM, Roa J, Luque RM et al. (2009) KiSS-1/ kisspeptins and the metabolic control of reproduction: physiologic roles and putative physiopathological implications. Peptides 30, 139-145.

48. Escrich E (1998) Endocrine aspects of breast cancer. In Senology, pp. 599-603 [J Cardoso, editor]. Bologna: Monduzzi Editore.

49. Hilakivi-Clarke L, Clarke R, Onojafe I et al. (1997) A maternal diet high in $\mathrm{n}-6$ polyunsaturated fats alters mammary gland development, puberty onset, and breast cancer risk among female rat offspring. Proc Natl Acad Sci USA 94, 9372-9377.

50. de Assis S, Khan G \& Hilakivi-Clarke L (2006) High birth weight increases mammary tumorigenesis in rats. Int $J$ Cancer 119, 1537-1546.

51. Stark AH, Kossoy G, Zusman I et al. (2003) Olive oil consumption during pregnancy and lactation in rats influences mammary cancer development in female offspring. Nutr Cancer 46, 59-65.

52. Sylvester PW, Russell M, Ip MM et al. (1986) Comparative effects of different animal and vegetable fats fed before and during carcinogen administration on mammary tumorigenesis, sexual maturation, and endocrine function in rats. Cancer Res 46, 757-762.

53. Russo IH \& Russo J (1996) Mammary gland neoplasia in long-term rodent studies. Environ Health Perspect 104, 938-967.

54. Escrich E, Moral R, Grau L et al. (2007) Molecular mechanisms of the effects of olive oil and other dietary lipids on cancer. Mol Nutr Food Res 51, 1279-1292.

55. Ortega RM (2006) Importance of functional foods in the Mediterranean diet. Public Health Nutr 9, 1136-1140.

56. Hulbert AJ, Turner N, Storlien LH et al. (2005) Dietary fats and membrane function: implications for metabolism and disease. Biol Rev Camb Philos Soc 80, 155-169.

57. Barceló F, Perona JS, Prades J et al. (2009) Mediterraneanstyle diet effect on the structural properties of the erythrocyte cell membrane of hypertensive patients: the Prevencion con Dieta Mediterranea Study. Hypertension 54, 1143-1150.

58. Jump DB (2004) Fatty acid regulation of gene transcription. Crit Rev Clin Lab Sci 41, 41-78.

59. Moral R, Solanas M, Manzanares EM et al. (2004) Influence of DMBA-induced mammary cancer on the liver CPT I, mit HMG-CoA synthase and PPAR $\alpha$ mRNA expression in rats fed low or high corn oil diet. Int J Mol Med 14, $283-287$.

60. Konstantinidou V, Covas MI, Muñoz-Aguayo D et al. (2010) In vivo nutrigenomic effects of virgin olive oil polyphenols within the frame of the Mediterranean diet: a randomized controlled trial. FASEB J 24, 2546-2557.

61. Malaney S \& Daly RJ (2001) The ras signaling pathway in mammary tumorigenesis and metastasis. J Mammary Gland Biol Neoplasia 6, 101-113.

62. Solanas M, Grau L, Moral R et al. (2010) Dietary olive oil and corn oil differentially affect experimental breast cancer through distinct modulation of the p21ras signaling and the proliferation-apoptosis balance. Carcinogenesis $\mathbf{3 1}$, 871-879.

63. Singh RP, Kumar R \& Kapur N (2003) Molecular regulation of cholesterol biosynthesis: implications in carcinogenesis. J Environ Pathol Toxicol Oncol 22, 75-92.

64. Moral R, Solanas M, Garcia G et al. (2008) High corn oil and high extra virgin olive oil diets have different effects on the expression of differentiation-related genes in experimental mammary tumors. Oncol Rep 20, 429-435.

65. Escrich E, Moral R, García G et al. (2004) Identification of novel differentially expressed genes by the effect of a highfat $\mathrm{n}-6$ diet in experimental breast cancer. Mol Carcinog $\mathbf{4 0}$, 73-78.

66. Solanas M, Moral R, Garcia G et al. (2009) Differential expression of H19 and vitamin D3 upregulated protein 1 as a mechanism of the modulatory effects of high virgin olive oil and high corn oil diets on experimental mammary tumours. Eur J Cancer Prev 18, 153-161.

67. Rao CV, Newmark HL \& Reddy BS (1998) Chemopreventive effect of squalene on colon cancer. Carcinogenesis 19, 287-290.

68. Franke TF, Hornik CP, Segev L et al. (2003) PI3K/Akt and apoptosis: size matters. Oncogene 22, 8983-8998. 
69. Hardy S, St-Onge GG, Joly E et al. (2005) Oleate promotes the proliferation of breast cancer cells via the $G$ proteincoupled receptor GPR40. J Biol Chem 280, 13285-13291.

70. Menendez JA, Vellon L, Colomer R et al. (2005) Oleic acid, the main monounsaturated fatty acid of olive oil, suppresses Her-2/neu (erbB-2) expression and synergistically enhances the growth inhibitory effects of trastuzumab (Herceptin) in breast cancer cells with Her-2/neu oncogene amplification. Ann Oncol 16, 359-371.

71. Guichard C, Pedruzzi E, Fay M et al. (2006) Dihydroxyphenylethanol induces apoptosis by activating serine/ threonine protein phosphatase PP2A and promotes the endoplasmic reticulum stress response in human colon carcinoma cells. Carcinogenesis 27, 1812-1827.

72. Chang GC, Hsu SL, Tsai JR et al. (2004) Extracellular signalregulated kinase activation and $\mathrm{Bcl}-2$ downregulation mediate apoptosis after gemcitabine treatment partly via a p53-independent pathway. Eur J Pharmacol 502, 169-183.
73. Karmakar S, Banik NL \& Ray SK (2008) Combination of all-trans retinoic acid and paclitaxel-induced differentiation and apoptosis in human glioblastoma U87MG xenografts in nude mice. Cancer 112, 596-607.

74. Jin Z \& El-Deiry WS (2005) Overview of cell death signaling pathways. Cancer Biol Ther 4, 139-163.

75. Han J, Talorete TP, Yamada P et al. (2009) Anti-proliferative and apoptotic effects of oleuropein and hydroxytyrosol on human breast cancer MCF-7 cells. Cytotechnology 59, 45-53.

76. Hardy S, Langelier Y \& Prentki M (2000) Oleate activates phosphatidylinositol 3-kinase and promotes proliferation and reduces apoptosis of MDA-MB-231 breast cancer cells, whereas palmitate has opposite effects. Cancer Res $\mathbf{6 0}$, 6353-6358.

77. Llor X, Pons E, Roca A et al. (2003) The effects of fish oil, olive oil, oleic acid and linoleic acid on colorectal neoplastic processes. Clin Nutr 22, 71-79. 